\title{
A New Approach to Infrared Microspectroscopy: Adding FT-IR to a Light Microscope
}

\author{
John A. Reffner, Donald K. Wilks, Kenneth C. Schreiber and Robert V. Burch \\ SensIR Technologies, Danbury CT 06810
}

A new infrared spectrometer and special objectives are described, which attach to a commercial light microscope, transforming the microscope into an infrared microprobe. When an infrared spectrometer is combined with a light microscopy a system is formed that can analyze molecular materials at the microscopic scale. In the past, a specialized microscope attached to a Fourier transform infrared (FT-IR) spectrometer was used in many spectroscopy laboratories. The new infrared microprobe technology combines a specialized FT-IR spectrometer with a commercial light microscope. This FT-IR spectrometer attachment is analogous to the energy dispersive X-ray (EDX) spectrometer attachment used on a scanning electron microscope. However, EDX detects elements while infrared detects molecular bonds; these are complementary not competitive techniques.

The infrared microprobe is a versatile instrument for analyzing molecular materials. Today, infrared microprobe analysis is accepted by spectroscopists as a routine technique for polymer science, trace evidence examinations, probing biological tissues and many other analyses of molecular materials. This marriage of photon (light) microscopy with molecular spectroscopy has stood the test of time and grows stronger year by year. However, unlike EDX spectroscopy, the microscopy community's acceptance of molecular spectroscopy lags behind; not leading the development but forcing spectroscopists to become microscopists. Now that a simple attachment can transform a light microscope into an infrared microprobe, microscopists may at last accept molecular spectroscopy as a vital tool.

A new interferometer was developed to meet the specific requirements of the infrared microprobe. The diameter of the objective pupil of most modern infinity corrected light microscopes is 20 to 22$\mathrm{mm}$. Samples for infrared microprobe analysis are either solids or liquids. For these condensed phases, the maximum spectral resolution $\left(v_{\max }\right)$ required is $4-\mathrm{cm}^{-1}$. For a Michelson interferometer to achieve this level of spectral resolution, the moving mirror must be translated only $1 /\left(2 v_{\max }\right)$. This means that the total mirror motion, allowing for mirror turn around, is less than 2-mm. The maximum analytical spot size was set at 100-um in diameter. This translates to an infrared source size of $0.25-\mathrm{mm}$ in diameter. Considering all of these factors, a small, robust, and efficient interferometer was designed. The interferometer is optically coupled to the microscope using a dichroic beamsplitter, which reflects the mid-infrared radiation and transmits visible light. The interferometer is imposed between the body of the microscope and its trinocular viewer as shown in Figure 1. Special infrared objectives are needed. Two new objectives are part of the IlluminatIR ${ }^{\mathrm{TM}}$ product, an all-reflecting objective for external reflection and a diamond objective for attenuated internal reflection. With these two objectives, any sample may be analyzed with little or no sample preparation. Thin samples are mounted on infrared reflective glass or metal slides in order to collect transmission spectra with the all-reflection objective. The diamond ATR objective, see Figure 2., is used for collecting spectra of either thick or thin samples. A major advantage of the ATR method is that it eliminates the need to prepare thin samples. An example of analyzing a fiber is shown in Figure 3. 
The microscope functions are unchanged by inserting the IlluminatIR in the light path and all standard viewing methods, such as fluorescence, DIC and polarized light can be used. Infrared spectral analysis extends the light microscope as EDX has extended the usefulness of the SEM.

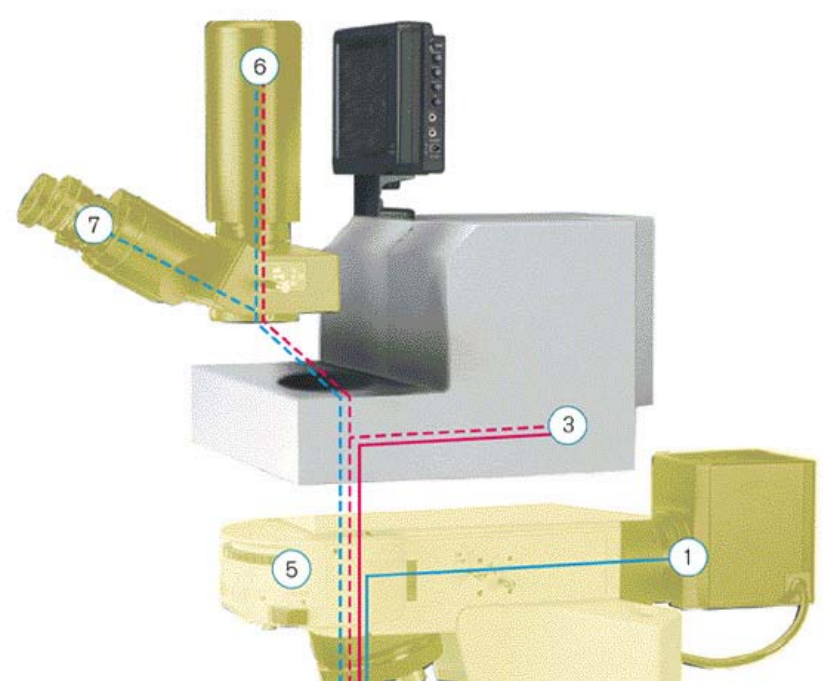

Figure 1. The IlluminatIR attachment
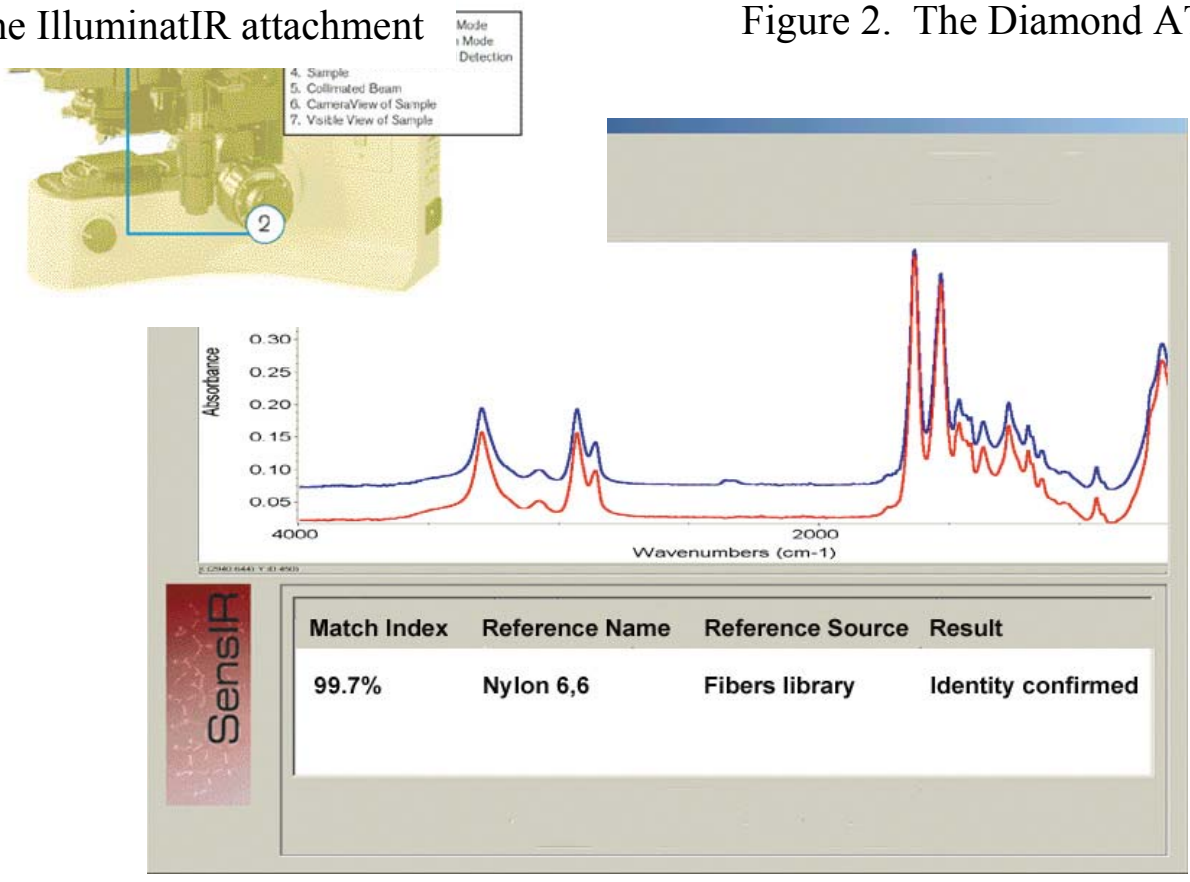

Figure 3. Fiber analysis
Figure 2. The Diamond ATR objective 